\title{
TWISTED POINCARÉ DUALITY FOR POISSON HOMOLOGY AND COHOMOLOGY OF AFFINE POISSON ALGEBRAS
}

\author{
CAN ZHU
}

(Communicated by Birge Huisgen-Zimmermann)

\begin{abstract}
This paper investigates the Poisson (co)homology of affine Poisson algebras. It is shown that there is a twisted Poincaré duality between their Poisson homology and cohomology. The relation between the Poisson (co)homology of an affine Poisson algebra and the Hochschild (co)homology of its deformation quantization is also discussed, which is similar to Kassel's result (1988) for homology and is a special case of Kontsevich's theorem (2003) for cohomology.
\end{abstract}

\section{INTRODUCTION}

Given a Poisson algebra, deformation quantization seeks a noncommutative algebra whose noncommutativity encodes the Poisson bracket on the previous Poisson algebra. There are deep relations between the Poisson (co)homology of a Poisson algebra with the Hochschild (co)homology of its deformation quantization. Brylinski's spectral sequence Bry88 and HKR-theorem [HKR62 link the Poisson homology of a Poisson algebra with the Hochschild homology of its deformation quantization, and also provide a way to compute the Hochschild homology of some algebras VdB94, Mar04, BP14. For the algebra of smooth functions over $\mathbb{R}^{n}$, Kontsevich proved that its Poisson cohomology is isomorphic to the Hochschild cohomology of the corresponding deformation as graded algebras Kon03. In 1999, Xu obtained a Poincaré duality between Poisson cohomology and Poisson homology for a unimodular Poisson manifold Xu99. Van den Bergh duality establishes a twisted Poincaré duality between Hochschild cohomology and Hochschild homology for some Gorenstein algebras VdB98. Calabi-Yau algebras are important ones which satisfy Van den Bergh duality Gin06. Recently, Dolgushev showed that the deformation quantization algebra, of the algebra of functions on a Poisson variety, is a Calabi-Yau algebra if and only if the corresponding Poisson structure is unimodular Dol09.

There are also many nonunimodular Poisson algebras and it would be interesting to search Xu's duality in a general sense. Such a duality was constructed in ELW99, Hue99] in an abstract way. By dualizing the complexes for computing the Poisson (co)homology, Launois and Richard proposed a version of Xu's duality for some quadratic Poisson algebras [LR07]. Using similar methods, in this paper we will study this duality for affine Poisson algebras.

Received by the editors January 16, 2013 and, in revised form, November 14, 2013.

2010 Mathematics Subject Classification. Primary 17B63, 18G60, 16 S30.

Key words and phrases. Affine Poisson algebra, Poisson (co)homology, Poincaré duality, enveloping algebra, Hochschild (co)homology. 
Let $V$ be a finite dimensional vector space and $S(V)$ be the symmetric algebra of $V$. A Poisson structure on $S(V)$ is called affine if the Poisson bracket is of degree $\leq 1$ where the grading on $S(V)$ is given by setting $\operatorname{deg} x=1$ for each $x \in V$ (see LPV13 for details). Let $\mathfrak{g}$ be a Lie algebra of dimension $n$ over the field $\mathbb{k}$, and let $f \in Z^{2}(\mathfrak{g}, \mathbb{k})$ be an arbitrary 2-cocycle, that is, $f: \mathfrak{g} \times \mathfrak{g} \rightarrow \mathbb{k}$ satisfying that

$$
f(x, x)=0 \quad \text { and } \quad f(x,[z, y])+f(y,[z, x])+f(z,[x, y])=0
$$

for any $x, y, z \in \mathfrak{g}$. Then, there is a Poisson structure on the symmetric algebra $S(\mathfrak{g})$ with the Poisson bracket induced by

$$
\{x, y\}=[x, y]+f(x, y)
$$

for $x, y \in \mathfrak{g}([\operatorname{Kas} 88])$. Here, $[x, y]$ is of degree 1 , and $f(x, y)$ of degree 0 in $S(\mathfrak{g})$. Hence, this is an affine Poisson structure. There is an associative algebra $U_{f}(\mathfrak{g})=$ $T_{\mathbb{k}}(\mathfrak{g}) / I$, where $I$ is the two-sided ideal generated by the elements

$$
x \otimes y-y \otimes x-[x, y]-f(x, y) .
$$

This algebra was introduced by Sridharan Sri61] and is often called Sridharan enveloping algebra. It is easy to see that Sridharan enveloping algebra is a deformation quantization of the above Poisson algebra (also a PBW deformation [Sri61]). The universal enveloping algebras and Weyl algebras are two important classes of Sridharan enveloping algebra. Moreover, according to [Sri61, Kas88, LPV13, any affine Poisson algebra can be achieved by Sridharan enveloping algebra of a Lie algebra $\mathfrak{g}$ and a 2-cocycle $f$.

Unless in general settings, we fix an $n$-dimensional Lie algebra $\mathfrak{g}$ and $f$ is an arbitrary 2-cocycle, $S=S(\mathfrak{g})$ is the Poisson algebra with the Poisson bracket (1.1) and $A$ is the Sridharan enveloping algebra $U_{f}(\mathfrak{g})$. Denote by $\nu$ the Nakayama automorphism of $A$. The main result of this paper reads

Theorem. There exists a Poisson module $S_{\nu}$ such that there is a twisted Poincaré duality between the Poisson homology of $S$ with coefficients in $S_{\nu}$ and the cohomology of $S$,

$$
\operatorname{HP}_{k}\left(S, S_{\nu}\right) \cong \operatorname{HP}^{n-k}(S), \forall k \in \mathbb{N} .
$$

The relation between the Poisson (co)homology of an affine Poisson algebra and the Hochschild (co)homology of its deformation quantization is discussed.

Theorem. For each $k \in \mathbb{N}$,

$$
\begin{aligned}
& \operatorname{HH}_{k}\left(A, A^{\nu}\right) \cong \operatorname{HP}_{k}\left(S, S_{\nu}\right), \\
& \mathrm{HH}^{k}(A) \cong \operatorname{HP}^{k}(S) .
\end{aligned}
$$

The paper is organized as follows. First, we retrieve the Nakayama automorphism and Van den Bergh duality for Sridharan enveloping algebras by a direct approach. Next, we recall some basic materials on Poisson algebras and their (co)homology, and construct a Poisson module providing a twisted Poincaré duality between Poisson homology and cohomology. In the last section, we study the relation between the Poisson (co)homology of an affine Poisson algebra and the Hochschild (co)homology of its deformation quantization.

Throughout the paper, $\mathbb{k}$ is a base field of characteristic 0 and all algebras are $\mathbb{k}$-algebras; unadorned $\otimes$ means $\otimes_{\mathbb{k}}$. 


\section{VAN DEN BERGH DUALITY}

Yekutieli [Ye00, and He, Van Oystaeyen and Zhang HVZ10] compute the rigid dualizing complex for universal and Sridaharan enveloping algebras. Then, it results in a twisted Poincaré duality between the Hochschild cohomology and homology from a theorem of Van den Bergh VdB98. In this section we reprove it by computing the Nakayama automorphism. We denote by $\operatorname{HH}_{*}(A, N)$ the Hochschild homology of an algebra $A$ with coefficients in the $A^{e}$-module $N$, and by $\operatorname{HH}_{*}(A)$ the Hochschild homology of an algebra $A$. We use similar notations for the Hochschild cohomologies.

Proposition 2.1 ([Kas88]). Suppose that $A$ is as in the introduction. Let $L_{k}(A):=$ $A \otimes \bigwedge^{k} \mathfrak{g} \otimes A$ and $d_{k}: L_{k}(A) \rightarrow L_{k-1}(A)$ be defined by

$$
\begin{aligned}
d_{k}\left(a \otimes x_{1} \wedge \cdots \wedge\right. & \left.x_{k} \otimes b\right):=\sum_{1 \leq i \leq k}(-1)^{i+1} a x_{i} \otimes x_{1} \wedge \cdots \wedge \widehat{x}_{i} \wedge \cdots \wedge x_{k} \otimes b \\
& -\sum_{1 \leq i \leq k}(-1)^{i+1} a \otimes x_{1} \wedge \cdots \wedge \widehat{x_{i}} \wedge \cdots \wedge x_{k} \otimes x_{i} b \\
& +\sum_{1 \leq i<j \leq k}(-1)^{i+j} a \otimes\left[x_{i}, x_{j}\right] \wedge x_{1} \wedge \cdots \wedge \widehat{x_{i}} \wedge \cdots \wedge \widehat{x_{j}} \wedge \cdots \wedge x_{k} \otimes b
\end{aligned}
$$

for any $a, b \in A$ and $x_{1}, \cdots, x_{k} \in \mathfrak{g}$. Then $\left(L_{*}(A), d_{*}\right)$ is a projective resolution of $A$ as an $A^{e}$-module.

When $f=0$, it is just the Chevalley-Eilenberg resolution for a universal enveloping algebra (see [CE56], for example).

Proposition 2.2. Let $A$ be as in the introduction. Then,

$$
\operatorname{Ext}_{A^{e}}^{i}\left(A, A^{e}\right) \cong \begin{cases}0, & i \neq n, \\ A^{\nu}, & i=n,\end{cases}
$$

as $A^{e}$-modules, where $\nu$ is an automorphism of $A$ induced by $\nu(x)=x+\operatorname{tr}(\operatorname{ad} x)$ for any $x \in \mathfrak{g}$, and $A^{\nu}$ denotes the $A^{e}$-module $A$ with the action

$$
(a \otimes b) \rightsquigarrow x:=a x \nu(b) .
$$

Such an automorphism is called a Nakayama automorphism in terms of [BZ08].

Proof. Applying the functor $\operatorname{Hom}_{A^{e}}\left(-, A^{e}\right)$ to the sequence $\left(L_{*}(A), d_{*}\right)$ and by using the filtration on this complex which is induced by the filtration of $A$, we obtain a spectral sequence with $E_{1}^{p, q}=\operatorname{Ext}_{(\operatorname{gr} A)^{e}}^{p+q}\left(\operatorname{gr} A,(\operatorname{gr} A)^{e}\right)_{p}$ the homogeneous part of degree $p$ of the cohomology group $\operatorname{Ext}_{(\operatorname{gr} A)^{e}}^{p+q}\left(\operatorname{gr} A,(\operatorname{gr} A)^{e}\right)$, and $E_{\infty}^{n}=$ $\operatorname{Ext}_{A^{e}}^{p+q}\left(A, A^{e}\right)$. It is known that gr $A$ is isomorphic to the symmetric algebra $S(\mathfrak{g})$. Hence, $\operatorname{Ext}_{A^{e}}^{i}\left(A, A^{e}\right)=0$ for $i \neq n$. Next, we compute the cohomology group $\operatorname{Ext}_{A^{e}}^{n}\left(A, A^{e}\right)$. First, for each finite dimensional $\mathbb{k}$-space $V$, there is an isomorphism

$$
\theta: A \otimes V^{*} \otimes A \rightarrow \operatorname{Hom}_{A^{e}}\left(A \otimes V \otimes A, A^{e}\right)
$$

as $A^{e}$-modules, where $V^{*}$ denotes the dual as a $\mathbb{k}$-space and $\theta(a \otimes \alpha \otimes b): c \otimes x \otimes d \mapsto$ $\alpha(x) c b \otimes a d$ for $\alpha \in V^{*}, x \in V$ and $a, b, c, d \in A$. Note that $\bigwedge^{n-1} \mathfrak{g}$ is a $\mathbb{k}$-space of dimension $n$ and so is $\left(\bigwedge^{n-1} \mathfrak{g}\right)^{*}$. We choose $\left\{x_{1}, \cdots, x_{n}\right\}$ as a basis for the $\mathbb{k}$-algebra $\mathfrak{g}$. Then, $\left\{x_{1} \wedge \cdots \wedge \widehat{x_{i}} \wedge \cdots \wedge x_{n}, i=1, \cdots, n\right\}$ is a basis for $\wedge^{n-1} \mathfrak{g}$. Denote $\left\{\left(\widehat{x_{i}}\right)^{*}, i=1, \cdots, n\right\}$ as the basis for the dual space $\left(\bigwedge^{n-1} \mathfrak{g}\right)^{*}$. Similarly, 
$\bigwedge^{n} \mathfrak{g}$ is a $\mathbb{k}$-space of dimension 1 with the base element $x_{1} \wedge \cdots \wedge x_{n}$ and denote $X$ for the base element of its dual space. Now, there is a commutative diagram

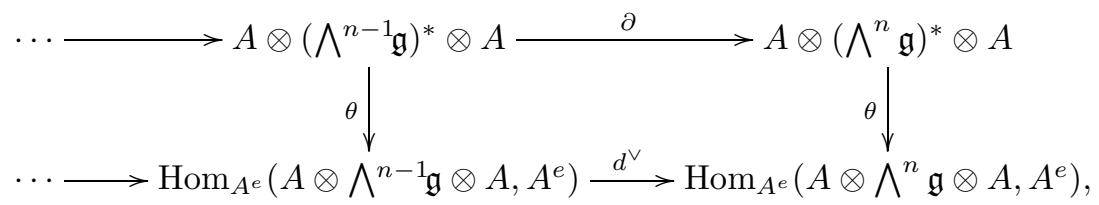

where $d^{\vee}$ represents the morphism $\operatorname{Hom}_{A^{e}}\left(d, A^{e}\right)$ and $\partial$ is given by

$$
\partial\left(a \otimes\left(\widehat{x_{k}}\right)^{*} \otimes b\right):=(-1)^{k}\left(a x_{k} \otimes X \otimes b-a \otimes X \otimes x_{k} b+\operatorname{tr}\left(\operatorname{ad} x_{k}\right) a \otimes X \otimes b\right) .
$$

Define $\rho: A \otimes\left(\bigwedge^{n} \mathfrak{g}\right)^{*} \otimes A \rightarrow A^{\nu}$ by $a \otimes X \otimes b \mapsto a \nu(b)$. Then, $\rho \partial=0$. That is, we have a complex of $A^{e}$-modules

$$
A \otimes\left(\bigwedge^{n-1} \mathfrak{g}\right)^{*} \otimes A \stackrel{\partial}{\rightarrow} A \otimes\left(\bigwedge^{n} \mathfrak{g}\right)^{*} \otimes A \stackrel{\rho}{\rightarrow} A^{\nu} \rightarrow 0 .
$$

By "filtration-gradation" techniques (see p. 288 Theorem 3 [NVO82, for example) and by the fact that $\operatorname{Ext}_{(\operatorname{gr} A)^{e}}^{n}\left(\operatorname{gr} A,(\operatorname{gr} A)^{e}\right)=\operatorname{gr} A$, the above sequence is exact. Therefore, $\operatorname{Ext}_{A^{e}}^{n}\left(A, A^{e}\right)=A^{\nu}$, which finishes the proof.

By using [VdB98, Theorem 1], we have the following

Proposition 2.3 (Van den Bergh duality). Let $A$ be as in the introduction. For every $A^{e}$-module $N$ and $i \in \mathbb{Z}$, there is a functorial isomorphism

$$
\operatorname{HH}^{i}(A, N) \cong \operatorname{HH}_{n-i}\left(A, A^{\nu} \otimes N\right) .
$$

Especially, there is a twisted Poincaré duality between the Hochschild cohomology and homology

$$
\mathrm{HH}^{i}(A) \cong \mathrm{HH}_{n-i}\left(A, A^{\nu}\right)
$$

\section{Twisted Poincaré DUality for Poisson homology and COHOMOlOGy}

In this section, we consider the Poisson (co)homology for affine Poisson algebras. We will construct a Poisson module and prove the main result. First, we recall some preliminaries including Poisson algebra, Poisson module and its (co)homology.

3.1. Poisson algebra and Poisson module. By definition a Poisson algebra is a commutative $\mathbb{k}$-algebra $S$ equipped with a bilinear map $\{-,-\}: S \otimes S \rightarrow S$ (Poisson bracket) satisfying

(1) $\{-,-\}$ is a Lie bracket,

(2) $\{-,-\}$ is a derivation in each variable.

Definition 3.2 (Oh99). A right Poisson module over the Poisson algebra $S$ is a $\mathbb{k}$-vector space $M$ endowed with two bilinear maps $\cdot$ and $\{-,-\}_{M}: M \otimes S \rightarrow M$ such that

(1) $(M, \cdot)$ is a right module over the commutative algebra $S$,

(2) $\left(M,\{-,-\}_{M}\right)$ is a right module over the Lie algebra $(S,\{-,-\})$,

(3) $\{x a, b\}_{M}=\{x, b\}_{M} \cdot a+x \cdot\{a, b\}$ for all $a, b \in S$ and $x \in M$,

(4) $\{x, a b\}_{M}=\{x, a\}_{M} \cdot b+\{x, b\}_{M} \cdot a$ for all $a, b \in S$ and $x \in M$. 
The left Poisson module is defined in a similar way. For a Poisson algebra $S$, the space $S$ has a natural right Poisson module structure. Now, let $S$ be as in the introduction. Let $S_{\nu}=S(\mathfrak{g})$ as vector space endowed with the following two actions:

- . is just the multiplication of $S$,

- $\{-,-\}_{S_{\nu}}: S_{\nu} \otimes S \rightarrow S_{\nu}$ is defined by

$$
\left\{m, x_{i}\right\}_{S_{\nu}}:=\left\{m, x_{i}\right\}+\operatorname{tr}\left(\operatorname{ad} x_{i}\right) m
$$

for all $m \in S_{\nu}$ and $i=1, \cdots, n$, where $\left\{m, x_{i}\right\}$ is the Poisson bracket in $S$. Then, $\left(S_{\nu}, \cdot,\{-,-\}_{S_{\nu}}\right)$ is a right Poisson module over $S$. As an $S$-module, $S_{\nu}$ is just $S$. But as a Poisson module, it differs from the right Poisson module $S$ in general. Here, we can see the Poisson module structure on $S_{\nu}$ is similar to the twisted module $A^{\nu}$ appearing in the Van den Bergh duality.

3.3. Poisson homology and cohomology. Let $M$ be a right Poisson module over a Poisson algebra $S$. Then there is a chain complex on the $S$-module $M \otimes_{S} \Omega^{k}(S)$ (see [Mas06]), where $\Omega^{k}(S)$ denotes the Kähler differential $k$-forms. The boundary operator $\partial_{k}: M \otimes_{S} \Omega^{k}(S) \rightarrow M \otimes_{S} \Omega^{k-1}(S)$ is defined by

$$
\begin{aligned}
& \partial_{k}\left(m \otimes d a_{1} \wedge \cdots \wedge d a_{k}\right)=\sum_{1 \leq i \leq k}(-1)^{i+1}\left\{m, a_{i}\right\}_{M} \otimes d a_{1} \wedge \cdots \widehat{d a_{i}} \wedge \cdots \wedge d a_{k} \\
& \quad+\sum_{1 \leq i<j \leq k}(-1)^{i+j} m \otimes d\left\{a_{i}, a_{j}\right\} \wedge d a_{1} \wedge \cdots \wedge \widehat{d a_{i}} \wedge \cdots \wedge \widehat{d a_{j}} \wedge \cdots \wedge d a_{k},
\end{aligned}
$$

where $d$ denotes the exterior differential. It is easy to see that $\partial_{k}$ is well defined and that $\partial_{k-1} \partial_{k}=0$. The homology of this complex is denoted by $\operatorname{HP}_{*}(S, M)$ and is called the Poisson homology of the Poisson algebra $S$ with coefficients in the Poisson module $M$.

Denote by $\chi^{k}(S)$ the space of all skew-symmetric $\mathbb{k}$-linear maps $S^{\otimes k} \rightarrow S$ that are derivations in each argument. Here, a map $f: S^{k} \rightarrow S$ is called skew-symmetric if $f\left(a_{1}, \cdots, a_{k}\right)=\varepsilon(\sigma) f\left(a_{\sigma_{1}}, \cdots, a_{\sigma_{k}}\right)$ for any permutation $\sigma \in S_{k}$, where $\varepsilon(\sigma)$ denotes its sign and $\sigma_{i}$ means $\sigma(i)$. Then there is a cochain complex $\left(\chi^{*}(S), \delta_{*}\right)$ ([Lic77, Hue90]), where $\delta_{k}: \chi^{k}(S) \rightarrow \chi^{k+1}(S)$ is defined by

$$
\begin{aligned}
\delta_{k}(P)\left(y_{0}, y_{1}, \cdots, y_{k}\right) & :=\sum_{0 \leq i \leq k}(-1)^{i}\left\{y_{i}, P\left(y_{0}, y_{1}, \cdots, \widehat{y}_{i}, \cdots, y_{k}\right)\right\} \\
& +\sum_{0 \leq i<j \leq k}(-1)^{i+j} P\left(\left\{y_{i}, y_{j}\right\}, y_{0}, y_{1}, \cdots, \widehat{y_{i}}, \cdots \widehat{y_{j}}, \cdots, y_{k}\right)
\end{aligned}
$$

for all $P \in \chi^{k}(S)$. It is not hard to check that $\delta_{k}(P)$ belongs to $\chi^{k+1}(S)$ and that $\delta_{k+1} \delta_{k}=0$. The cohomology of this complex is denoted by $\operatorname{HP}^{*}(S)$ and is called the Poisson cohomology of the Poisson algebra $S$.

3.4. Twisted Poincaré duality. Let $S$ be as in the introduction. It is known that the set $\chi^{k}(S)$ is isomorphic as a vector space to $\Omega^{n-k}(S)$ via an isomorphism $\dagger$ defined as follows. Denote by $S_{k, n-k}$ the set of $(k, n-k)$-shuffle, i.e., those permutations $\sigma \in S_{n}$ such that $\sigma_{1}<\sigma_{2}<\cdots<\sigma_{k}$ and $\sigma_{k+1}<\sigma_{k+2}<\cdots<\sigma_{n}$. Then, for any $P \in \chi^{k}(S)$ let $\dagger(P)$ be the unique element of $\Omega^{n-k}(S)$ defined by

$$
\dagger(P)=\sum_{\sigma \in S_{k, n-k}} \varepsilon(\sigma) P\left(x_{\sigma_{1}}, x_{\sigma_{2}}, \cdots, x_{\sigma_{k}}\right) d x_{\sigma_{k+1}} \wedge \cdots \wedge d x_{\sigma_{n}} .
$$


Since $S_{\nu}=S$ as a vector space the map $\dagger$ will induce an isomorphism between the vector spaces $\chi^{k}(S)$ and $S_{\nu} \otimes_{S} \Omega^{n-k}(S)$. Now, we have the following diagram:

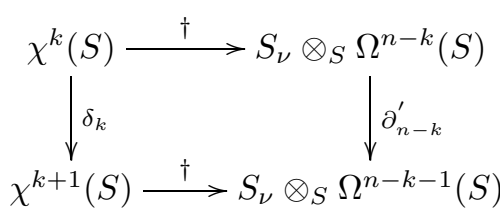

where the map $\partial^{\prime}$ stands for id $\otimes \partial$. First, we prove the following.

Proposition 3.5. For any $P \in \chi^{k}(S)$, the following equality holds:

$$
\dagger \delta(P)=(-1)^{k+1} \partial^{\prime} \dagger(P) .
$$

Proof. First, it follows from the definition that $\dagger \delta(P)=U_{1}+V_{1}$, where

$$
U_{1}:=\sum_{\substack{\sigma \in S_{k+1, n-k-1} \\ 1 \leq i \leq k+1}} \varepsilon(\sigma)(-1)^{i+1}\left\{x_{\sigma_{i}}, P\left(x_{\sigma_{1}}, \cdots, \widehat{x_{\sigma_{i}}}, \cdots, x_{\sigma_{k+1}}\right)\right\} d x_{\sigma_{k+2}} \wedge \cdots \wedge d x_{\sigma_{n}}
$$

and

$$
\begin{gathered}
V_{1}:=\sum_{\substack{\sigma \in S_{k+1, n-k-1} \\
1 \leq i<j \leq k+1}} \varepsilon(\sigma)(-1)^{i+j} P\left(\left\{x_{\sigma_{i}}, x_{\sigma_{j}}\right\}, x_{\sigma_{1}}, \cdots, \widehat{x_{\sigma_{i}}}, \cdots, \widehat{x_{\sigma_{j}}}, \cdots, x_{\sigma_{k+1}}\right) \\
\otimes d x_{\sigma_{k+2}} \wedge \cdots \wedge d x_{\sigma_{n}} .
\end{gathered}
$$

Since $S_{\nu}$ is a Poisson module, we have

$$
\begin{aligned}
& \partial^{\prime} \dagger(P)=\sum_{\sigma \in S_{k, n-k}} \varepsilon(\sigma)\left(\sum_{i=1}^{n-k}(-1)^{i+1}\left\{P\left(x_{\sigma_{1}}, \cdots, x_{\sigma_{k}}\right), x_{\sigma_{k+i}}\right\}_{S_{\nu}} d x_{\sigma_{k+1}} \wedge \cdots \widehat{d x_{\sigma_{k+i}}} \cdots \wedge d x_{\sigma_{n}}\right. \\
& +\sum_{1 \leq i<j \leq n-k}(-1)^{i+j} P\left(x_{\sigma_{1}}, \cdots, x_{\sigma_{k}}\right) d\left\{x_{\sigma_{k+i}}, x_{\sigma_{k+j}}\right\} \\
& \left.\wedge d x_{\sigma_{k+1}} \wedge \cdots \widehat{d x_{\sigma_{k+i}}} \cdots \widehat{d x_{\sigma_{k+j}}} \cdots \wedge d x_{\sigma_{n}}\right) \\
& =\sum_{\sigma \in S_{k, n-k}} \varepsilon(\sigma)\left(\sum_{i=1}^{n-k}(-1)^{i+1}\left\{P\left(x_{\sigma_{1}}, \cdots, x_{\sigma_{k}}\right), x_{\sigma_{k+i}}\right\} d x_{\sigma_{k+1}} \wedge \cdots \wedge \widehat{d x_{\sigma_{k+i}}} \wedge \cdots \wedge d x_{\sigma_{n}}\right. \\
& +\sum_{i=1}^{n-k}(-1)^{i+1} \operatorname{tr}\left(\operatorname{ad} x_{\sigma_{i+k}}\right) P\left(x_{\sigma_{1}}, \cdots, x_{\sigma_{k}}\right) d x_{\sigma_{k+1}} \wedge \cdots \wedge \widehat{d x_{\sigma_{k+i}}} \wedge \cdots \wedge d x_{\sigma_{n}} \\
& \left.+\sum_{1 \leq i<j \leq n-k}(-1)^{i+j} P\left(x_{\sigma_{1}}, \cdots, x_{\sigma_{k}}\right) d\left\{x_{\sigma_{k+i}}, x_{\sigma_{k+j}}\right\} \wedge d x_{\sigma_{k+1}} \cdots \widehat{d x_{\sigma_{k+i}}} \cdots \widehat{d x_{\sigma_{k+j}}} \cdots \wedge d x_{\sigma_{n}}\right) \text {. }
\end{aligned}
$$

Denote the first term by $U_{2}$, and let $V_{2}$ be the sum of the last two terms on the right-hand side in the above equality.

We will present this in four steps.

Step 1. We rewrite $U_{1}$. For $r$ distinct integers $i_{1}, \cdots, i_{r}$, let $\left(i_{1}, \cdots, i_{r}\right)$ be the cyclic permutation sending $i_{1}$ to $i_{2}, \cdots, i_{r-1}$ to $i_{r}$ and $i_{r}$ to $i_{1}$. Let $S_{k, 1, n-k-1}$ be the set of those permutations $\tau \in S_{n}$ with $\tau_{1}<\tau_{2}<\cdots<\tau_{k}$ and $\tau_{k+2}<$ $\tau_{k+2}<\cdots<\tau_{n}$. Then, the map $(\sigma, i) \in S_{k+1, n-k-1} \times\{1,2, \cdots, k+1\} \mapsto \tau:=$ $\sigma \circ(i, i+1, \cdots, k+1) \in S_{k, 1, n-k-1}$ is well defined and is a bijection. Moreover, 
one has $\varepsilon(\tau)=(-1)^{k+1-i} \varepsilon(\sigma)$. Hence, by the changing of variables induced by this bijection, we have

$$
U_{1}=(-1)^{k} \sum_{\tau \in S_{k, 1, n-k-1}} \varepsilon(\tau)\left\{x_{\tau_{k+1}}, P\left(x_{\tau_{1}}, \cdots, x_{\tau_{k}}\right)\right\} d x_{\tau_{k+2}} \wedge \cdots \wedge d x_{\tau_{n}}
$$

Step 2. We rewrite $V_{1}$. First, note that $P\left(y_{1}, \cdots, y_{k}\right)=0$ if there exists $i \neq j$ such that $y_{i}=y_{j}$ or if some $y_{i} \in k$ by definition. Suppose that $\left[x_{i}, x_{j}\right]:=\sum_{1 \leq l \leq n} a_{l}^{i, j} x_{l}$; then $\left\{x_{i}, x_{j}\right\}=\sum_{1 \leq l \leq n} a_{l}^{i, j} x_{l}+f\left(x_{i}, x_{j}\right)$. Obviously, $a_{l}^{i, j}=-a_{l}^{j, i}$ by the antisymmetry of the Poisson bracket. Then,

$$
\begin{aligned}
& V_{1}:=\sum_{\substack{\sigma \in S_{k+1, n-k-1} \\
1 \leq i<j \leq k+1}} \varepsilon(\sigma)(-1)^{i+j} P\left(\sum_{1 \leq l \leq n} a_{l}^{\sigma_{i}, \sigma_{j}} x_{l}, x_{\sigma_{1}}, \cdots, \widehat{x_{\sigma_{i}}}, \cdots, \widehat{x_{\sigma_{j}}}, \cdots, x_{\sigma_{k+1}}\right) \\
& \otimes d x_{\sigma_{k+2}} \wedge \cdots \wedge d x_{\sigma_{n}} \\
& =\sum_{\substack{\sigma \in S_{k+1, n-k-1} \\
1 \leq i<j \leq k+1}} \varepsilon(\sigma)(-1)^{i+j} P\left(a_{\sigma_{i}}^{\sigma_{i}, \sigma_{j}} x_{\sigma_{i}}, x_{\sigma_{1}}, \cdots, \widehat{x_{\sigma_{i}}}, \cdots, \widehat{x_{\sigma_{j}}}, \cdots, x_{\sigma_{k+1}}\right) \\
& \otimes d x_{\sigma_{k+2}} \wedge \cdots \wedge d x_{\sigma_{n}} \\
& +\sum_{\substack{\sigma \in S_{k+1, n-k-1} \\
1 \leq i<j \leq k+1}} \varepsilon(\sigma)(-1)^{i+j} P\left(a_{\sigma_{j}}^{\sigma_{i}, \sigma_{j}} x_{\sigma_{j}}, x_{\sigma_{1}}, \cdots, \widehat{x_{\sigma_{i}}}, \cdots, \widehat{x_{\sigma_{j}}}, \cdots, x_{\sigma_{k+1}}\right) \\
& \otimes d x_{\sigma_{k+2}} \wedge \cdots \wedge d x_{\sigma_{n}} \\
& =\sum_{\sigma \in S_{k+1, n-k-1}} \varepsilon(\sigma) \sum_{i=1}^{k}(-1)^{i} \sum_{j=i+1}^{k+1} a_{\sigma_{j}}^{\sigma_{i}, \sigma_{j}} P\left(x_{\sigma_{1}}, \cdots, \widehat{x_{\sigma_{i}}}, \cdots, x_{\sigma_{k+1}}\right) \\
& \otimes d x_{\sigma_{k+2}} \wedge \cdots \wedge d x_{\sigma_{n}} \\
& +\sum_{\sigma \in S_{k+1, n-k-1}} \varepsilon(\sigma) \sum_{j=2}^{k+1}(-1)^{j+1} \sum_{i=1}^{j-1} a_{\sigma_{i}}^{\sigma_{i}, \sigma_{j}} P\left(x_{\sigma_{1}}, \cdots, \widehat{x_{\sigma_{j}}}, \cdots, x_{\sigma_{k+1}}\right) \\
& \otimes d x_{\sigma_{k+2}} \wedge \cdots \wedge d x_{\sigma_{n}} \\
& =\sum_{\sigma \in S_{k+1, n-k-1}} \varepsilon(\sigma) \sum_{i=1}^{k}(-1)^{i} \sum_{j=i+1}^{k+1} a_{\sigma_{j}}^{\sigma_{i}, \sigma_{j}} P\left(x_{\sigma_{1}}, \cdots, \widehat{x_{\sigma_{i}}}, \cdots, x_{\sigma_{k+1}}\right) \\
& \otimes d x_{\sigma_{k+2}} \wedge \cdots \wedge d x_{\sigma_{n}} \\
& +\sum_{\sigma \in S_{k+1, n-k-1}} \varepsilon(\sigma) \sum_{i=2}^{k+1}(-1)^{i+1} \sum_{j=1}^{i-1} a_{\sigma_{j}}^{\sigma_{j}, \sigma_{i}} P\left(x_{\sigma_{1}}, \cdots, \widehat{x_{\sigma_{i}}}, \cdots, x_{\sigma_{k+1}}\right) \\
& \otimes d x_{\sigma_{k+2}} \wedge \cdots \wedge d x_{\sigma_{n}} \\
& =\sum_{\sigma \in S_{k+1, n-k-1}} \varepsilon(\sigma) \sum_{i=1}^{k+1}(-1)^{i} \sum_{j=1}^{k+1} a_{\sigma_{j}}^{\sigma_{i}, \sigma_{j}} P\left(x_{\sigma_{1}}, \cdots, \widehat{x_{\sigma_{i}}}, \cdots, x_{\sigma_{k+1}}\right) \otimes \\
& \otimes d x_{\sigma_{k+2}} \wedge \cdots \wedge d x_{\sigma_{n}} .
\end{aligned}
$$


Then, using the same technique as in the previous step, we get

$$
V_{1}=\sum_{\tau \in S_{k, 1, n-k-1}} \varepsilon(\tau)(-1)^{k+1} \sum_{j=1}^{k} a_{\tau_{j}}^{\tau_{k+1}, \tau_{j}} P\left(x_{\tau_{1}}, \cdots, x_{\tau_{k}}\right) \otimes d x_{\tau_{k+2}} \wedge \cdots \wedge d x_{\tau_{n}} .
$$

Step 3. By a similar progress as in Step 1, one can easily get

$$
U_{2}=\sum_{\tau \in S_{k, 1, n-k-1}} \varepsilon(\tau)\left\{P\left(x_{\tau_{1}}, \cdots, x_{\tau_{k}}\right), x_{\tau_{k+1}}\right\} d x_{\tau_{k+2}} \wedge \cdots \wedge d x_{\tau_{n}}
$$

Hence, $U_{1}=(-1)^{k+1} U_{2}$.

Step 4. We rewrite $V_{2}$ and conclude. Note that $\operatorname{tr}\left(\operatorname{ad} x_{i}\right)=\sum_{1 \leq l \leq n} a_{l}^{i, l}$. To simplify the notation, write $Q$ for $P\left(x_{\sigma_{1}}, \cdots, x_{\sigma_{k}}\right)$. We obtain

$$
\begin{aligned}
& V_{2}=\sum_{\sigma \in S_{k, n-k}} \varepsilon(\sigma)\left(\sum_{i=1}^{n-k}(-1)^{i+1} \operatorname{tr}\left(\operatorname{ad} x_{\sigma_{i+k}}\right) Q d x_{\sigma_{k+1}} \wedge \cdots \wedge \widehat{d x_{\sigma_{k+i}}} \wedge \cdots \wedge d x_{\sigma_{n}}\right. \\
& +\sum_{1 \leq i<j \leq n-k}(-1)^{i+j} Q d\left(\sum_{1 \leq l \leq n} a_{l}^{\sigma_{k+i}, \sigma_{k+j}} x_{l}+f\left(x_{i}, x_{j}\right)\right) \\
& \left.\wedge d x_{\sigma_{k+1}} \wedge \cdots \widehat{d x_{\sigma_{k+i}}} \cdots \widehat{d x_{\sigma_{k+j}}} \cdots \wedge d x_{\sigma_{n}}\right) \\
& =\sum_{\sigma \in S_{k, n-k}} \varepsilon(\sigma)\left(\sum_{i=1}^{n-k}(-1)^{i+1} \operatorname{tr}\left(\operatorname{ad} x_{\sigma_{i+k}}\right) Q d x_{\sigma_{k+1}} \wedge \cdots \wedge \widehat{d x_{\sigma_{k+i}}} \wedge \cdots \wedge d x_{\sigma_{n}}\right. \\
& +\sum_{i=1}^{n-k-1} \sum_{j=i+1}^{n-k}(-1)^{j+1} a_{\sigma_{i+k}}^{\sigma_{k+i}, \sigma_{k+j}} Q d x_{\sigma_{k+1}} \wedge \cdots \wedge \widehat{d x_{\sigma_{k+j}}} \wedge \cdots \wedge d x_{\sigma_{n}} \\
& \left.+\sum_{j=2}^{n-k} \sum_{i=1}^{j-1}(-1)^{i} a_{\sigma_{j+k}}^{\sigma_{k+i}, \sigma_{k+j}} Q d x_{\sigma_{k+1}} \wedge \cdots \wedge \widehat{d x_{\sigma_{k+i}}} \wedge \cdots \wedge d x_{\sigma_{n}}\right) \\
& =\sum_{\sigma \in S_{k, n-k}} \varepsilon(\sigma)\left(\sum_{i=1}^{n-k}(-1)^{i+1} \sum_{l=1}^{n} a_{l}^{\sigma_{k+i}, l} Q d x_{\sigma_{k+1}} \wedge \cdots \wedge \widehat{d x_{\sigma_{k+i}}} \wedge \cdots \wedge d x_{\sigma_{n}}\right. \\
& \left.+\sum_{i=1}^{n-k} \sum_{j=1}^{n-k}(-1)^{i} a_{\sigma_{j+k}}^{\sigma_{k+i}, \sigma_{k+j}} Q d x_{\sigma_{k+1}} \wedge \cdots \wedge \widehat{d x_{\sigma_{k+i}}} \wedge \cdots \wedge d x_{\sigma_{n}}\right) \\
& =\sum_{\sigma \in S_{k, n-k}} \varepsilon(\sigma) \sum_{i=1}^{n-k}(-1)^{i+1} \sum_{l=1}^{k} a_{\sigma_{l}}^{\sigma_{k+i}, \sigma_{l}} Q d x_{\sigma_{k+1}} \wedge \cdots \wedge \widehat{d x_{\sigma_{k+i}}} \wedge \cdots \wedge d x_{\sigma_{n}} \\
& =\sum_{\tau \in S_{k, 1, n-k-1}} \varepsilon(\tau) \sum_{l=1}^{k} a_{\tau_{l}}^{\tau_{k+i}, \tau_{l}} P\left(x_{\tau_{1}}, \cdots, x_{\tau_{k}}\right) d x_{\tau_{k+1}} \wedge \cdots \wedge \widehat{d x_{\tau_{k+i}}} \wedge \cdots \wedge d x_{\tau_{n}} .
\end{aligned}
$$

Thus, $V_{1}=(-1)^{k+1} V_{2}$. We must point out that in Step 3 and Step 4 we use the bijection map $(\sigma, i) \in S_{k, n-k} \times\{1,2, \cdots, n-k\} \mapsto \tau:=\sigma \circ(k+i, k+i-1, \cdots, k+1) \in$ $S_{k, 1, n-k}$ and $\varepsilon(\tau)=(-1)^{i-1} \varepsilon(\sigma)$.

Therefore, we obtain that $\dagger \delta(P)=(-1)^{k+1} \partial^{\prime} \dagger(P)$.

Now, the diagram considered is commutative up to sign. As a consequence, the following theorem is proved. 
Theorem 3.6. For all $k \in \mathbb{N}$, we have $\operatorname{HP}_{k}\left(S, S_{\nu}\right) \cong \operatorname{HP}^{n-k}(S)$.

\section{Application}

Suppose that an algebra $A$ has a filtration such that the associated graded algebra $\operatorname{gr} A$ is commutative and smooth. Brylinski introduced a spectral sequence $E^{1}:=$ $\mathrm{HH}_{*}(\mathrm{gr} A) \Longrightarrow \mathrm{HH}_{*}(A)$ in Bry88. Moreover, by HKR-theorem HKR62, the $E^{2}$ page of this spectral sequence is equal to the Poisson homology. Kassel showed that when the associated graded algebra is a symmetric algebra, this spectral sequence degenerates. Therefore, $\mathrm{HH}_{*}(A) \cong \mathrm{HP}_{*}(S)$ ([Kas88]). The following is similar to Kassel's result.

Proposition 4.1. Let $A$ and $S$ be as in the introduction. Then, for all $k \in \mathbb{N}$, we have

$$
\mathrm{HH}_{k}\left(A, A^{\nu}\right) \cong \operatorname{HP}_{k}\left(S, S_{\nu}\right)
$$

Proof. Applying the functor $A^{\nu} \otimes_{A^{e}}-$ to the resolution in Proposition 2.1 and by the canonical isomorphism $A^{\nu} \otimes_{A^{e}}(A \otimes V \otimes A) \cong A^{\nu} \otimes V$, the Hochschild homology $\mathrm{HH}_{*}\left(A, A^{\nu}\right)$ will be equal to the homology group of the following chain complex:

$$
\cdots \rightarrow A^{\nu} \otimes \bigwedge^{k} \mathfrak{g} \stackrel{b^{\prime}}{\rightarrow} A^{\nu} \otimes \bigwedge^{k-1} \mathfrak{g} \rightarrow \cdots \rightarrow A^{\nu},
$$

where the boundary $b^{\prime}$ is

$$
\begin{aligned}
b^{\prime}\left(a \otimes x_{1} \wedge \cdots \wedge x_{k}\right):=\sum_{1 \leq i \leq k}(-1)^{i+1}\left(a \nu\left(x_{i}\right)-x_{i} a\right) \otimes x_{1} \wedge \cdots \wedge \widehat{x_{i}} \wedge \cdots \wedge x_{k} \\
\quad+\sum_{1 \leq i<j \leq k}(-1)^{i+j} a \otimes\left[x_{i}, x_{j}\right] \wedge x_{1} \wedge \cdots \wedge \widehat{x_{i}} \wedge \cdots \wedge \widehat{x_{j}} \cdots \wedge x_{k} .
\end{aligned}
$$

Recall that $\nu$ is an automorphism of $A$ induced by $\nu(x)=x+\operatorname{tr}(\operatorname{ad} x)$ for any $x \in \mathfrak{g}$. Hence, $a \nu\left(x_{i}\right)-x_{i} a=a x_{i}-x_{i} a+\operatorname{tr}\left(\operatorname{ad} x_{i}\right) a$.

Obviously, the $S$-module of the Kähler differential $k$-forms $\Omega^{k}(S)$ is isomorphic to $S \otimes \bigwedge^{k} \mathfrak{g}$ as $S$-modules. Hence, the Poisson homology group $\operatorname{HP}_{*}\left(S, S_{\nu}\right)$ will be equal to the homology group of the following chain complex:

$$
\cdots \rightarrow S_{\nu} \otimes \bigwedge^{k} \mathfrak{g} \stackrel{\delta^{\prime}}{\rightarrow} S_{\nu} \otimes \bigwedge^{k-1} \mathfrak{g} \rightarrow \cdots \rightarrow S_{\nu}
$$

where the boundary $\delta^{\prime}$ is

$$
\begin{aligned}
\delta^{\prime}\left(a \otimes x_{1} \wedge \cdots \wedge x_{k}\right):=\sum_{1 \leq i \leq k}(-1)^{i+1}\left\{a, x_{i}\right\}_{S_{\nu}} \otimes x_{1} \wedge \cdots \wedge \widehat{x_{i}} \wedge \cdots \wedge x_{k} \\
\quad+\sum_{1 \leq i<j \leq k}(-1)^{i+j} a \otimes\left[x_{i}, x_{j}\right] \wedge x_{1} \wedge \cdots \wedge \widehat{x_{i}} \wedge \cdots \wedge \widehat{x_{j}} \cdots \wedge x_{k} .
\end{aligned}
$$

Next, we claim that the following diagram is commutative:

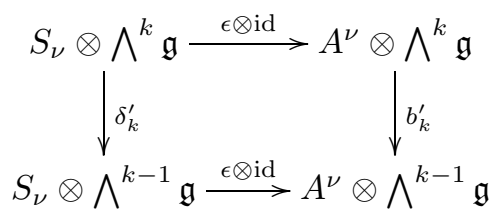


where $\epsilon: S \rightarrow A$ is an isomorphism of $\mathbb{k}$-vector space by the Poincaré-Birkhoff-Witt theorem and is defined by

$$
\epsilon\left(x_{1} x_{2} \cdots x_{m}\right):=\frac{1}{m !} \sum_{\sigma \in S_{m}} x_{\sigma_{1}} x_{\sigma_{2}} \cdots x_{\sigma_{m}} .
$$

According to Kas88, Lemma 6], the equation

$$
\epsilon\left(\left\{g, x_{i}\right\}\right)=\epsilon(g) x_{i}-x_{i} \epsilon(g)
$$

holds in $A$ for all $g \in S$ and $x_{i} \in \mathfrak{g}$. Then, the diagram is commutative and we get the desired conclusion.

In Kon03, Kontsevich proved that the Poisson cohomology of the algebra of smooth functions over $\mathbb{R}^{n}$ is isomorphic to the Hochschild cohomology of the corresponding deformation quantization. Here, we get the following which is a special case of Kontsevich's result.

Corollary 4.2. Let $A$ and $S$ be as above. Then, for each $k \in \mathbb{N}$ we have

$$
\mathrm{HH}^{k}(A) \cong \operatorname{HP}^{k}(S) .
$$

Proof. This is a direct consequence of Propositions 2.3 and 4.1 and Theorem 3.6 .

\section{ACKNOWLEDGMENTS}

This work was supported by the Natural Science Foundation of China (No. 11201299) and the Shanghai University Young Teacher Training Program (No. slg12024). The author thanks Professor Quan Shui Wu for his helpful comments and suggestions.

\section{REFERENCES}

[BP14] Roland Berger and Anne Pichereau, Calabi-Yau algebras viewed as deformations of Poisson algebras, Algebr. Represent. Theory 17 (2014), no. 3, 735-773, DOI 10.1007/s10468-013-9417-z. MR.3254768

[BZ08] K. A. Brown and J. J. Zhang, Dualising complexes and twisted Hochschild (co)homology for Noetherian Hopf algebras, J. Algebra 320 (2008), no. 5, 1814-1850, DOI 10.1016/j.jalgebra.2007.03.050. MR2437632(2009j:16007)

[Bry88] Jean-Luc Brylinski, A differential complex for Poisson manifolds, J. Differential Geom. 28 (1988), no. 1, 93-114. MR.950556 (89m:58006)

[CE56] Henri Cartan and Samuel Eilenberg, Homological algebra, Princeton University Press, Princeton, N. J., 1956. MR0077480 (17,1040e)

[Dol09] Vasiliy Dolgushev, The Van den Bergh duality and the modular symmetry of a Poisson variety, Selecta Math. (N.S.) 14 (2009), no. 2, 199-228, DOI 10.1007/s00029-008-0062-z. MR2480714 (2009m:53235)

[ELW99] Sam Evens, Jiang-Hua Lu, and Alan Weinstein, Transverse measures, the modular class and a cohomology pairing for Lie algebroids, Quart. J. Math. Oxford Ser. (2) 50 (1999), no. 200, 417-436, DOI 10.1093/qjmath/50.200.417. MR 1726784 (2000i:53114)

[Gin06] V. Ginzburg, Calabi-Yau algebras, arXiv: math. AG/0612139.

[HVZ10] Ji-Wei He, Fred Van Oystaeyen, and Yinhuo Zhang, Cocommutative Calabi-Yau Hopf algebras and deformations, J. Algebra 324 (2010), no. 8, 1921-1939, DOI 10.1016/j.jalgebra.2010.06.010. MR2678828(2011f:16079)

[HKR62] G. Hochschild, Bertram Kostant, and Alex Rosenberg, Differential forms on regular affine algebras, Trans. Amer. Math. Soc. 102 (1962), 383-408. MR0142598 (26 \#167)

[Hue90] Johannes Huebschmann, Poisson cohomology and quantization, J. Reine Angew. Math. 408 (1990), 57-113, DOI 10.1515/crll.1990.408.57. MR1058984 (92e:17027) 
[Hue99] Johannes Huebschmann, Duality for Lie-Rinehart algebras and the modular class, J. Reine Angew. Math. 510 (1999), 103-159, DOI 10.1515/crll.1999.043. MR.1696093 (2000f:53109)

[Kas88] Christian Kassel, L'homologie cyclique des algèbres enveloppantes (French, with English summary), Invent. Math. 91 (1988), no. 2, 221-251, DOI 10.1007/BF01389366. MR.922799 (89e:17015)

[Kon03] Maxim Kontsevich, Deformation quantization of Poisson manifolds, Lett. Math. Phys. 66 (2003), no. 3, 157-216, DOI 10.1023/B:MATH.0000027508.00421.bf. MR2062626 (2005i:53122)

[LPV13] Camille Laurent-Gengoux, Anne Pichereau, and Pol Vanhaecke, Poisson structures, Grundlehren der Mathematischen Wissenschaften [Fundamental Principles of Mathematical Sciences], vol. 347, Springer, Heidelberg, 2013. MR2906391

[LR07] Stéphane Launois and Lionel Richard, Twisted Poincaré duality for some quadratic Poisson algebras, Lett. Math. Phys. 79 (2007), no. 2, 161-174, DOI 10.1007/s11005006-0133-z. MR.2301394(2008d:17033)

[Lic77] André Lichnerowicz, Les variétés de Poisson et leurs algèbres de Lie associées (French), J. Differential Geometry 12 (1977), no. 2, 253-300. MR0501133 (58 \#18565)

[Mar04] Nicolas Marconnet, Homologies of cubic Artin-Schelter regular algebras, J. Algebra 278 (2004), no. 2, 638-665, DOI 10.1016/j.jalgebra.2003.11.019. MR2071658(2005m:16013)

[Mas06] T. Maszczyk, Maximal commutative subalgebras, Poisson geometry and Hochschild homology, arXiv: math.KT/0603386.

[NVO82] C. Năstăsescu and F. Van Oystaeyen, Graded ring theory, North-Holland Mathematical Library, vol. 28, North-Holland Publishing Co., Amsterdam, 1982. MR676974 (84i:16002)

[Oh99] Sei-Qwon Oh, Poisson enveloping algebras, Comm. Algebra 27 (1999), no. 5, 2181-2186, DOI 10.1080/00927879908826556. MR.1683858(2000b:16050)

[Sri61] R. Sridharan, Filtered algebras and representations of Lie algebras, Trans. Amer. Math. Soc. 100 (1961), 530-550. MR0130900 (24 \#A754)

[VdB94] Michel Van den Bergh, Noncommutative homology of some three-dimensional quantum spaces, Proceedings of Conference on Algebraic Geometry and Ring Theory in honor of Michael Artin, Part III (Antwerp, 1992), K-Theory 8 (1994), no. 3, 213-230, DOI 10.1007/BF00960862. MR.1291019 (95i:16009)

[VdB98] Michel Van den Bergh, A relation between Hochschild homology and cohomology for Gorenstein rings, Proc. Amer. Math. Soc. 126 (1998), no. 5, 1345-1348, DOI 10.1090/S0002-9939-98-04210-5. MR:1443171 (99m:16013)

[Xu99] Ping $\mathrm{Xu}$, Gerstenhaber algebras and BV-algebras in Poisson geometry, Comm. Math. Phys. 200 (1999), no. 3, 545-560, DOI 10.1007/s002200050540. MR.1675117 (2000b:17025)

[Ye00] Amnon Yekutieli, The rigid dualizing complex of a universal enveloping algebra, J. Pure Appl. Algebra 150 (2000), no. 1, 85-93, DOI 10.1016/S0022-4049(99)00032-8. MR 1762922 (2001g:16014)

College of Science, University of Shanghai for Science and Technology, Shanghai 200093, People's Republic of China

E-mail address: czhu@usst.edu.cn 